\title{
Aesthetic Quantification as Search Criteria in Architectural Design
}

\section{Archinder}

\author{
Victor Sardenberg ${ }^{1}$, Theron Burger ${ }^{2}$, Mirco Becker ${ }^{3}$ \\ 1,2,3 Leibniz Universität Hannover \\ ${ }^{1,2,3}$ \{sardenberg|burger|becker\}@aida.uni-hannover.de
}

The paper describes a research experiment of incorporating quantitative aesthetic evaluation and feeding the metric back into a parametric model to steer the search within the design space for a high-ranking design solution. The experiment is part of a longer-standing interest and research in quantitative aesthetics. $A$ web platform inspired by dating apps was developed to retrieve an aesthetic score of images (drawings and photographs of architectural projects). The app and scoring system was tested for functionality against an existing dataset of aesthetic measure (triangles, polygon nets). In the actual experiment, an evolutionary algorithm generated images of design candidates (phenotypes) and used the aesthetic score retrieved by the "crowd" of app users as a fitness function for the next generation/population. The research is in the tradition of empirical aesthetics of G. T. Fechner (Fechner, 1876), using a web app to crowdsource aesthetic scores and using these to evolve design candidates. The paper describes how the system is set up and presents its results in four distinct exercises.

Keywords: Quantitative Aesthetics, Social Media, Crowdsourcing, Collaborative Design, Human-Computer interaction

\section{INTRODUCTION - AESTHETIC QUANTIFI- CATION}

The research is presented in two parts: Firstly, it proposes a method for establishing a quantitative aesthetic measure. Secondly, it sets out the experiment where that measure is used to navigate the solution space of a parametric model.

At first, an aesthetic score for a set of images has to be established. This is done by recording the hedonic response individuals show towards a set of images. The mechanism is to present the images and record the responses to that in a social media app. A hedonic response is a judgement of liking or disliking something (Shimamura, 2014). One good example of an application of digitally enabled hedonic judgement is online dating apps like Tinder, where on one side you make an aesthetic judgement of liking or disliking photographs of other people, and by the other side, you share pictures of yourself to (hopefully) be liked by others. A system like that allows to translate a qualitative appreciation of images into a quantitative count of likes or dislikes: an aesthetic 
score.

When establishing an aesthetic measure, the research builds on the field of empirical aesthetics, which was established by the German philosopher, physicist and experimental psychologist Gustav Theodor Fechner (1801-1887). Fechner proposed a scientific method to evaluate human sensation that co-relates physical stimuli to it (Fechner, 1876). In this way, he avoided dealing with complicated philosophical questions and focused on how simple elements, like geometrical shapes and colours, produced effects on humans and how much they are liked in comparison with each other in a quantitative fashion.

There is a broad body of work on the question of why we appreciate some objects more than others with conclusions from different perspectives and from different disciplines, like psychology, neurology, philosophy, art history... However, the present work will focus on hedonic responses and not in the deeper aesthetic reasons.

The second part of the work is an experiment questioning how that measure could be fed back into a design process. We defend that besides structural, environmental and economic criteria, architecture could benefit also from an aesthetic evaluation when looking for design solutions. Antecedents application of a qualitative aesthetic-based search in the solution space are Sjoberg et. al that uses supervised Machine Learning to navigate the solution space (Sjoberg et. al, 2017) and Yusif et. al that incorporates form diversity and user interaction as parameters in Multi-Objective Genetic Algorithms (Yusif et. al, 2017). Other precedents are the research done at the "Brain Hacking" studio by Toru Hasegawa, Mark Collins and Anna Stork at Columbia GSAPP with eyetracking and EEG and a business application of quantitative aesthetics analysis is incorporated by Google to design its results pages (Granka et al. 2008).

One of the contributions of our proposed method is to implement social media web interfaces for crowdsourcing aesthetic evaluation. As a test group, 10 undergraduate architecture students coming from different countries used our mechanism. During one month, they fed our system with their hedonic evaluation and, in the end, used it for a design proposal.

\section{METHODS - SYSTEM SETUP AND TESTS}

Our system is separated in 3 elements:

1) Form Generating Parametric Model (FGPM)

2) Evolutionary Algorithm (EA)

3) Web Platform (WP)

The first element, FMPM, is open to any designer able to work with Rhinoceros and Grasshopper. It's a parametric model able to produce different designs according to a combination of parameters, therefore producing a design solution space. In the present paper, two different FMPM were developed by us and will be presented in the next chapters: (A) Single triangles and (B) Polygon nets. Also, the participants of this experiment developed their own FMPM for architectural elements, as presented in chapter D of this paper.

For the second part, an Evolutionary Algorithm (EA) was used to search the design space of the FMPM. Our EA inserts random values into the FMPM to create the first generation of design solutions and capture an image for each one. After receiving an aesthetic score of each solution from the Web Platform (WP), our EA creates new solutions tending towards the most liked previous images to create the next generations of drawings. The algorithm was run daily to upload newer images to the WP until it stabilizes, which means when the solutions are all too similar.

The last element of the system is the Web Platform (WP) with Graphic User Interface, a responsive web application with a custom server running on a cloud instance. It receives all images from all design solutions and presents it to the participants with a Graphic User Interface that mimics Tinder (Figure 1): It shows the image with a like or dislike button below and, also, allows the participant to swipe it left (dislike) or right (like). Every day the WP sends the aesthetic score to the EA, ranging from 0 (most disliked) 
to 1 (most liked).

Figure 1

Graphic User

Interface of

Archinder running

on a smartphone.

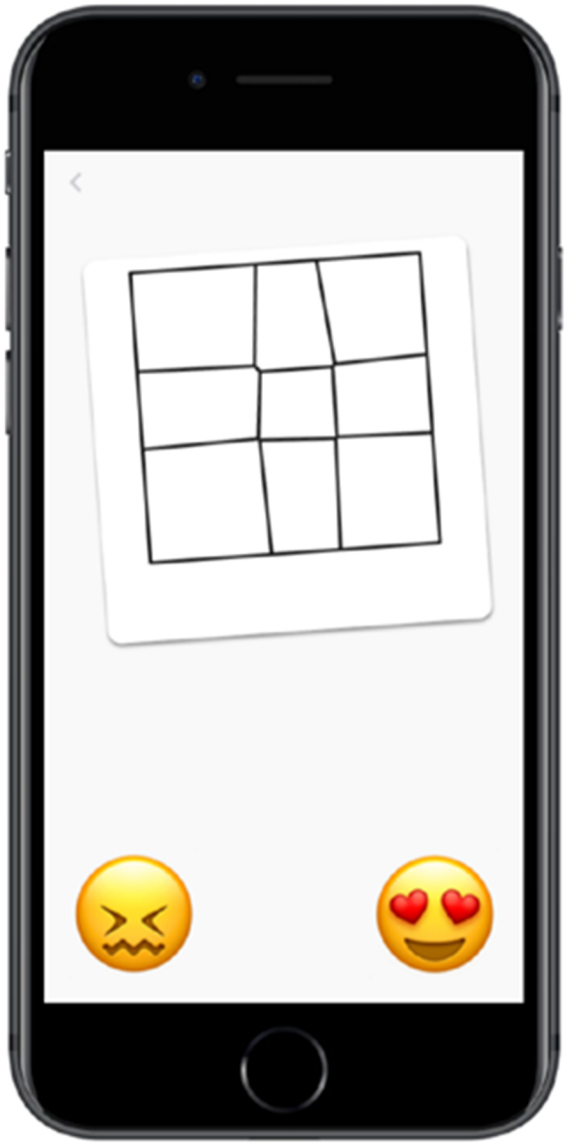

\section{RESULTS AND DISCUSSION}

The paper will present four outcomes from Archinder that deals with specific problems:

- A - Triangle Proportions (to test the overall functionality of the system),

- B - Polygon net (to evaluate complexity),

- C - Historical images (to add more image re- lated evaluation) and

- D - Architectural Elements (to allow participants to introduce their own designs).

\section{A - Triangle Proportions}

To determine if the whole system is working accordingly and to refer to existing research on perception, we realized a test based on established conclusions on the appreciation of symmetric triangles. Using hedonic responses, the state of the art research asserts that equilateral triangles receive more positive reactions than triangles with other proportions (Friedenberg, 2012). As Jay Friedenberg argues, "triangles that are more compact are less likely to move or break and are thus considered more pleasing".

From the first generation of randomly generated triangles, the system rapidly converged and produced equilateral triangles in the second generation. In the coming generations, the solutions became stable, not varying largely. It proved that the system was behaving as expected.

\section{B - Polygon Net. Between Boring and Dis- turbing: The problem of Complexity}

The second test focused on comparing the hedonic response of perceived simplicity and complexity. As a form making mechanism, the Voronoi diagram was adopted considering that it can produce complex geometry, simple grids and all sorts of variation between both

The first generation, that was randomly produced, exhibited simple grids and highly intricated polygons. The next two generations, fed by the data from our system, created solutions in between both extremes until it reached stability from the 4th generation onwards. This is a good example of the application of our system because Polygon Net operates with a very high number of variables.

In these images, complexity could be measured according to the variation of the size of cells and differences in the shape of each polygon. These results suggest that humans favour mild complexity.

Daniel Berlyne defends that humans are not at- 


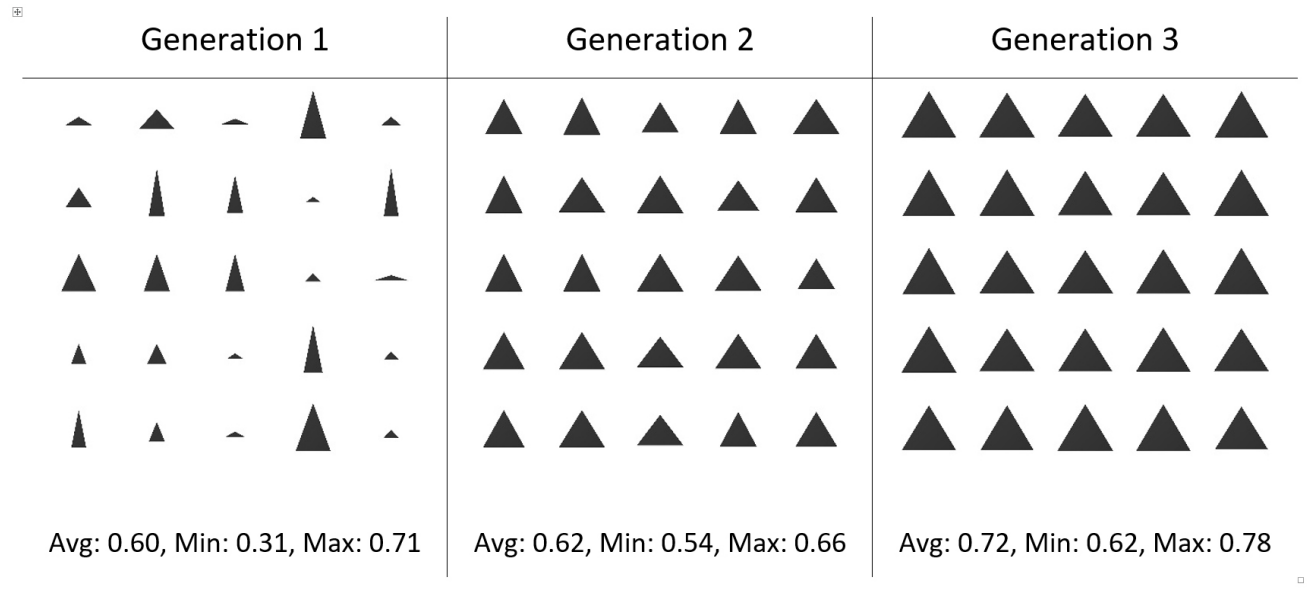

tracted by images that do little to arouse curiosity nor by works that are overpowering and causes confusion and displeasure (Berlyne, 1971). Berlyne argues that we prefer some novelty, surprise, complexity and incongruity but in excess, it causes a negative experience. Here it's important to note that this aesthetic theory is based on what we, as subjects, were previously exposed, defending that it's not an inborn mathematical proportion issue.

\section{C - The Problem of Images}

We decided in the next experiment to feed our system with existing pictures to avoid geometry and incorporate other qualities like colour, texture and shadows. Each student was asked to select their ten favourite buildings and upload one picture of each. In this case, we could only assert an aesthetic score to each according to the number of likes and dislikes, ranging from 0 (most disliked) to 1 (most liked). In this example the FMPM and the EA were by-passed.

It's not the ambition of this paper to make an aesthetic judgement of existing architectural projects, but it's important to note that a few images of the same building received very diverse aesthetical scores. Guggenheim in Bilbao appears twice and its lowest score is 0.380 and highest is 0.629 . The Pyra- mid of Khafre scored 0.476 and 0.714 .

\section{D - Aesthetic optimization applied in an Ar- chitectural Element}

To explore the application of the system, the participants developed their own FMPM of a vertical Architectural Element (Figure 2). All models were incorporated in the EA and uploaded to the WP.
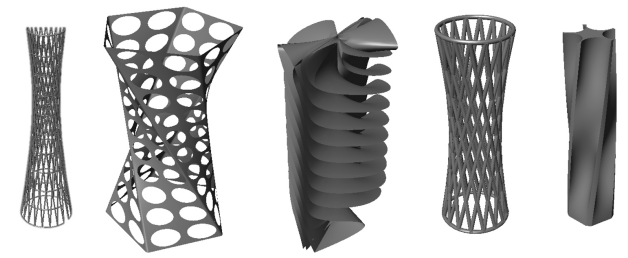

In this stage, the system is varying the parameters of each solution within each FMPM and also evaluating between all FMPM. After six generations, the solution with the highest aesthetic score was selected for further development.

This experiment proves that the system works to select architectural forms according to an aesthetic score but it demands more time to stabilize and, therefore, the designers should be aware to restrict the space of solutions. Evolutionary al-
Table 1

Three generations of triangles created by our Genetic Algorithm. Avg: Average Score, Min: Minimum Score, Max: Maximum Score

Figure 2

Five vertical architectural elements produced by five FMPM. 
Table 2

Six generations of polygon nets created by our Genetic Algorithm.
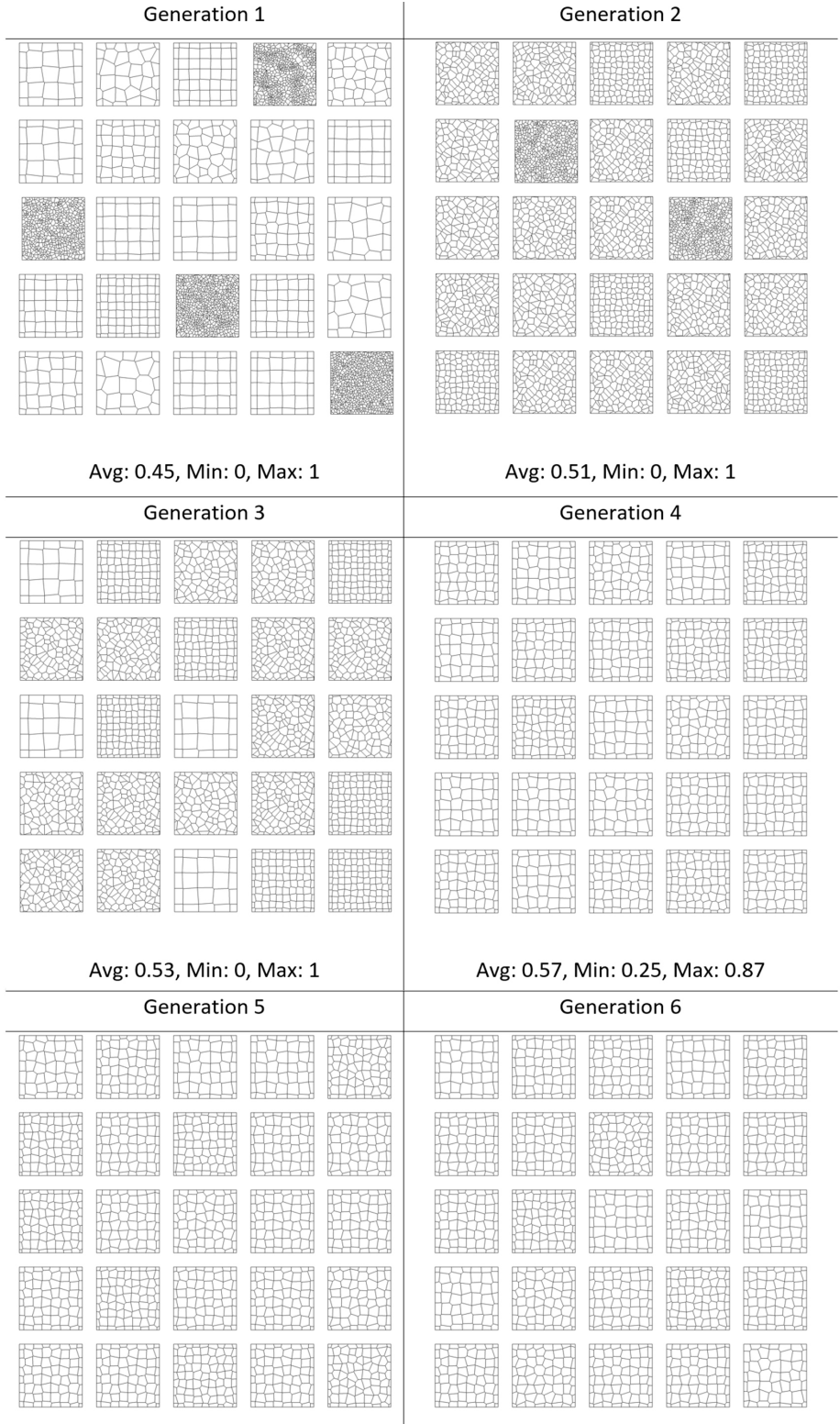

Avg: 0.65 , Min: 0.35 , Max: 0.91
Avg: 0.68, Min: 0.35, Max: 1 


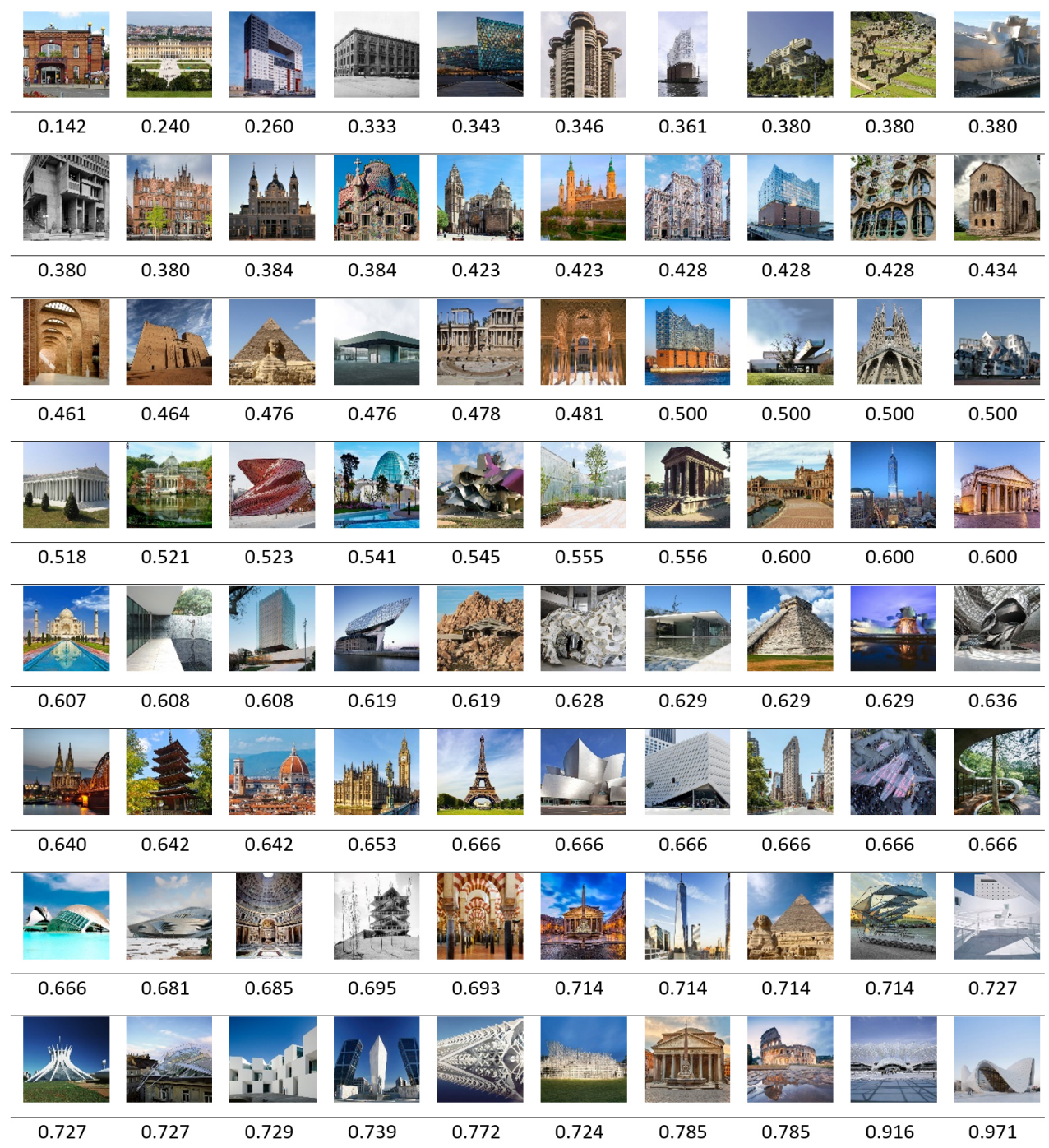

Table 3

80 photographs and its respective aesthetic score 
Table 4

Six generations of Architectural

Elements created by our Genetic Algorithm.

\begin{tabular}{|c|c|c|c|c|c|c|c|c|}
\hline \multicolumn{5}{|c|}{ Generation 1} & \multicolumn{4}{|c|}{ Generation 2} \\
\hline 型 & 8 & 糔 & 1 & 4 & 11 & 4 & 1 & 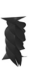 \\
\hline 輵 & I & 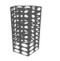 & 1 & $\nabla$ & II & 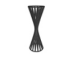 & 要 & 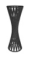 \\
\hline 1 & ) & 1 & 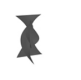 & I & 霖 1 & 麗 & 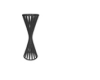 & 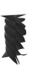 \\
\hline t & 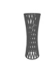 & 1 & 7 & I & 81 & 1 & 1 & 1 \\
\hline 8 & 要 & $\mathbf{I}$ & 1 & 数 & 7 & 羁 & & $\mathbf{I}$ \\
\hline \multicolumn{5}{|c|}{$\begin{array}{l}\text { Avg: } 0.65, \text { Min: } 0.11, \text { Max: } 0.91 \\
\text { Generation } 3\end{array}$} & \multicolumn{4}{|c|}{$\begin{array}{l}\text { Avg: } 0.48, \text { Min: } 0.09, \text { Max: } 0.78 \\
\text { Generation } 4\end{array}$} \\
\hline 4 & I & 1 & 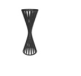 & 8 & 18 & 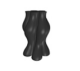 & 4 & 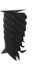 \\
\hline 8 & I & I & 8 & I & 麗 & 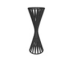 & 1 & 蜆 \\
\hline 4 & 灀 & I & 䊅 & I & 18 & 1 & 1 & I \\
\hline 4 & 管 & 】 & I & 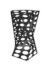 & $\nabla$ & 1 & 1 & | \\
\hline 1 & 8 & 8 & 璘 & 1 & 41 & 1 & 1 & I \\
\hline \multicolumn{5}{|c|}{$\begin{array}{c}\text { Avg: } 0.51 \text {, Min: } 0.14 \text {, Max: } 1 \\
\text { Generation } 5\end{array}$} & \multicolumn{4}{|c|}{$\begin{array}{c}\text { Avg: 0.57, Min: 0.19, Max: } 0.91 \\
\text { Generation } 6\end{array}$} \\
\hline$\sqrt{4}$ & 8 & 1 & I & I & 18 & 8 & 1 & 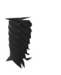 \\
\hline 4 & 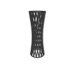 & 1 & 1 & 8 & 41 & 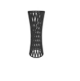 & 7 & I \\
\hline 軥 & 】 & I & 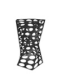 & 1 & & 8 & 1 & 8 \\
\hline 8 & 4 & 8 & 1 & 1 & 18 & $\mathbf{I}$ & $\searrow$ & 1 \\
\hline & 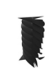 & 1 & 1 & 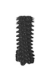 & 11 & 7 & & 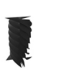 \\
\hline & & & & & & & Max: 1 & \\
\hline
\end{tabular}

Design - GENERATIVE SYSTEMS - Volume 1 - eCAADe 37 / SIGraDi 23 | 23 
gorithms in design are very time effective because usually the fitness criteria are calculated by the computer (like structural behaviour, environmental performance, cost estimation...), differing from our human-machine interface, that requires the test group to evaluate each solution.

\section{CONCLUSIONS}

The system proved to be a viable way to generate and select design solutions. In our time of big data and fast and cheap computers, it has become possible to generate and test an ever-increasing number of design solutions (Carpo, 2017). However, the criteria for the selection is usually a quantitative analysis that is closer to issues of engineering and economics than to architectural formal problems. Our system introduces the problem of aesthetics as possible criteria for design solution search.

The discrepancies in scores of two photographs of the same building in experiment $C$ with historical images made us realize that the object of analysis in our software is the aesthetic qualities of images themselves, being it drawings, renders of photographs and not necessarily the formal or spatial qualities of architectural projects. Therefore, the way that every image is generated is of primary importance and to compare design solutions it's essential to maintain visual consistency. Future work can go towards purely image evaluation not relying so much upon geometry.

One future development is to incorporate in the system the educational background of each user in order to compare how it influences the aesthetic judgement of images. It was already proven that the brain activity of educated architects and the general public are activated in different manners [7]. So, it will be interesting to see how architects' hedonic responses differ or not from the general public.

Another future development is to make it into a Grasshopper plug-in and make it available for the designer community to test the concept of aesthetic search in different environments, both in the academy and in practice and train Neural Networks according to the community's hedonic response.

\section{REFERENCES}

Berlyne, D 1971, Aesthetics and Psychobiology, AppletonCentury-Crofts

Carpo, M 2017, The Second Digital Turn: Design Beyond Intelligence, MIT Press

Fechner, GT 1876, Vorschule der Aesthetik, Breitkopf, Leipzig

Friedenberg, J 2012, 'Aesthetic judgment of triangular shape: compactness and not the golden ratio determines perceived attractiveness', i-Perception, 3(3), pp. $163-175$

Granka, L, Feusner, M and Lorigo, L 2008, 'Eye Monitoring in Online Search', in Hammoud, R (eds) 2008, Passive Eye Monitoring. Signals and Commmunication Technologies, Springer

Kirke, U 2014, 'The Modularity of Aesthetic Processing and Perception in the Human Brain: Functional Neuroimaging Studies of Neuroaesthetics', in Shimamura, AP and Palmer, SE (eds) 2014, Aesthetic Science, Oxford University Press

Shimamura, AP 2014, 'Towards a Science of Aesthetics: Issues and Ideas', in Shimamura, AP and Palmer, SE (eds) 2014, Aesthetic Science, Oxford University Press, pp. 3-28

Sjoberg, C, Beorkrem, C and Ellinger, J 2017 'Emergent Syntax: Machine Learning for the Curaton of Design Soluton Space', ACADIA 2017, pp. 552 - 561

Yousif, S, Yan, W and Culp, C 2017 'Incorporating Form Diversity into Architectural Design Optimization', $A C A-$ DIA 2017, pp. 640 - 649 